\title{
Resolving the hyper-triton yield description puzzle in high energy nuclear collisions
}

\author{
O. V. Vitiuk ${ }^{1,2}$, K. A. Bugaev ${ }^{1,3}$, E. S. Zherebtsova ${ }^{4,5}$, D. B. Blaschke $e^{4,6,7, a}$, L. V. Bravina ${ }^{2}$, E. E. Zabrodin ${ }^{2,8}$, \\ G. M. Zinovjev ${ }^{3}$ \\ ${ }^{1}$ Department of Physics, Taras Shevchenko National University of Kyiv, 03022 Kyiv, Ukraine \\ ${ }^{2}$ University of Oslo, POB 1048, Blindern, 0316 Oslo, Norway \\ ${ }_{3}^{3}$ Bogolyubov Institute for Theoretical Physics, Metrologichna str. 14B, Kyiv 03680, Ukraine \\ ${ }^{4}$ National Research Nuclear University (MEPhI), Kashirskoe Shosse 31, 115409 Moscow, Russia \\ ${ }^{5}$ Institute for Nuclear Research, Russian Academy of Science, 108840 Moscow, Russia \\ ${ }^{6}$ Institute of Theoretical Physics, University of Wroclaw, Max Born Pl. 9, 50-204 Wroclaw, Poland \\ ${ }^{7}$ Bogoliubov Laboratory of Theoretical Physics, JINR Dubna, Joliot-Curie Str. 6, 141980 Dubna, Russia \\ ${ }^{8}$ Skobeltsyn Institute of Nuclear Physics, Moscow State University, 119899 Moscow, Russia
}

Received: 28 July 2020 / Accepted: 29 December 2020 / Published online: 24 February 2021

(C) The Author(s) 2021

Communicated by Thomas Duguet

\begin{abstract}
The recently developed hadron resonance gas model with multicomponent hard-core repulsion is used to address and resolve the long standing problem to describe the light nuclear cluster multiplicities including the hyper-triton measured by the STAR Collaboration, known as the hypertriton chemical freeze-out puzzle. An improved description for the hadronic and light nuclear cluster data measured by STAR at the collision energy $\sqrt{s_{N N}}=200 \mathrm{GeV}$ and by ALICE at $\sqrt{s_{N N}}=2.76 \mathrm{TeV}$ is obtained. This is achieved by applying a new strategy of analyzing the light nuclear cluster data and by using the value for the hard-core radius of the (anti-) $\Lambda$ hyperons found in earlier work. One of the most striking results of the present work is that for the most probable scenario of chemical freeze-out for the STAR energy the obtained parameters allow to simultaneously reproduce the values of the experimental ratios $S_{3}$ and $\bar{S}_{3}$ which were not included in the fit.
\end{abstract}

\section{Introduction}

The yields of light (anti-) and (hyper-) nuclei, i.e. the light nuclear clusters, measured in heavy ion collisions at high energies triggered a vivid discussion about the proper theoretical formulation of the cluster formation process [1-4], since at the moment there are serious conceptual questions related to it. Such nuclei like the deuteron (d), helium-3 $\left({ }^{3} \mathrm{He}\right)$, helium-4 $\left({ }^{4} \mathrm{He}\right)$, hyper-triton $\left({ }_{\Lambda}^{3} \mathrm{H}\right)$ and their antipar-

\footnotetext{
a e-mail: david.blaschke@uwr.edu.pl (corresponding author)
}

ticles should be extremely sensitive to the assumptions of the model description, since their binding energies are much lower than the typical hadronic mass scales and the chemical freeze-out (CFO) temperature. Two major approaches with different underlying theoretical concepts, namely the coalescence models and the versions of the thermal hadron resonance gas model (HRGM), have been developed to describe the light nuclear cluster production in heavy-ion collisions, see, e.g., [5-10]. They are applied to describe the recent data, but not all of them are successfully reproduced $[1-4,10]$. In particular, the problematic hyper-triton ratios (PHTR) ${ }_{\Lambda}^{3} \overline{\mathrm{H}} /{ }^{3} \overline{\mathrm{He}}$ and ${ }_{\Lambda}^{3} \mathrm{H} /{ }^{3} \mathrm{He}$ measured by the STAR Collaboration at $\sqrt{s_{N N}}=200 \mathrm{GeV}[11,12]$ are not explained up to now.

In this work we suggest a simple, but elegant solution of the puzzle of the PHTR measured by the STAR Collaboration at $\sqrt{s_{N N}}=200 \mathrm{GeV}$. It allows us to simultaneously describe all hadronic and light nuclear cluster multiplicities which were obtained at this collision energy previously and very recently [13]. A similar analysis is performed for the ALICE LHC data [14-16] measured at $\sqrt{s_{N N}}=2.76 \mathrm{TeV}$. The newly developed HRGM [17-22] based on the induced surface tension (IST) equation of state (EoS) [23-27,34] allows us not only to achieve high quality description of hadronic and nuclear cluster yields and/or their ratios, but it also provides a new and high standard for HRGM existing nowadays.

Using this opportunity we would like to analyze the reasons why the other versions of HRGM fail to resolve the PHTR puzzle and to discuss what problems will face the 
HRGM community once the accuracy of measured hadronic and the light nuclear cluster yields will be improved by an order of magnitude in the future experiments on NICA JINR and FAIR GSI.

In addition we continue to refine the novel strategy to analyze the light nuclear cluster data which was first applied to the analysis of the ALICE data [14-16] measured at $\sqrt{s_{N N}}=2.76 \mathrm{TeV}$ in [21] and then it was successfully implemented to the analysis of the STAR data [11-13] in [22]. This strategy is based on the simultaneous use of two formulations of the IST EoS which, nevertheless, employ a different treatment of the hard-core repulsion. The first approach is based on the classical excluded volumes of light nuclear clusters with all hadrons found in [21] and it is, indeed, the IST EoS. The second, an approximative, but rather accurate approach called the bag model radii (BMR) EoS [22], employs an approximate treatment of the hard-core radii of light nuclear clusters in a pion dominated medium $[21,22]$. By construction both approaches should give the same quality of data description for some common values of CFO parameters. In this way one can remove the freedom in choosing the most probable hypothesis of CFO. Here we generalize such a strategy to arbitrary mixtures of hadrons with hard-core repulsion. As a result, the generalized strategy allows us to easily resolve the PHTR puzzle without spoiling the perfect description of the STAR and ALICE data mentioned above. Predictions for the yields of popular nuclear clusters and for some exotic nuclei are also made.

The work is organized as follows. In Sect. 2 we thoroughly discuss the necessary ingredients for the successful description of hadronic and light nuclear cluster yields and inspect the pitfalls of the other versions of HRGM. In addition in Sect. 2 the necessary mathematical apparatus is outlined. The analyses of the STAR and ALICE data mentioned above is given in Sect. 3, while the conclusions are summarized in Sect. 4.

\section{Main elements of IST-based HRGM EoS}

\subsection{Justification of multicomponent hard-core repulsion}

The success of advanced versions of the HRGM is based on a balance between the simple parameterization of the interaction among the hadrons and the detailed accounting for such properties of hadrons and hadronic resonances as their masses, charges, degeneracies, widths and decays into other hadrons. On the one hand, similarly to the hard-core repulsion among atoms [28,29], the one among hadrons reflects the fact that real hadrons are not point-like particles and the Pauli exclusion principle applied to the internal constituents of hadrons generates a repulsive interaction between them. In other words, the quarks and anti-quarks, belonging to dif- ferent hadrons, generate a very strong repulsion due to the Pauli blocking effect [30]. Note that very recently the validity of this qualitative picture was explored quantitatively in an explicit calculation of the chirally improved quark Pauli blocking effect among nucleons as three-quark bound states in nuclear matter [30] where also the corresponding nucleonic excluded volume parameters and hard-core radii were obtained. On the other hand, such a simple parameterization of hadronic interaction is well justified by the strong cancellation of the attractive and repulsive interaction [31] known from the HRGM with the quantum second virial coefficients of hadrons. Therefore, the residual deviation from the ideal gas with a weak repulsion can be taken into account as the hard-core repulsion via the classical second virial coefficients of hadrons.

It is important to stress that a sufficiently realistic EoS of hadronic matter with hard-core repulsion prevents the pressure in HRGM from exceeding the one of the quark-gluon plasma. The latter may happen, however, if the hadronic matter is treated as a mixture of ideal gases of all known hadrons and hadronic resonances [23,24,32-34]. Thus, the presence of hard-core repulsion provides an agreement with the simulations of lattice quantum chromodynamics (QCD) [35,36].

Moreover, an additional reason to consider the HRGM with hard-core repulsion as hadronic matter EoS in the vicinity of CFO is a purely practical one: since the hard-core repulsion is a contact interaction, the energy per particle of such an EoS coincides with the one of the ideal gas, even for the case of quantum statistics $[24,25]$. As a result, to model the evolution of the system created in heavy ion collisions between the CFO and the kinetic freeze-out one does not need to solve a hard mathematical problem $[37,38]$ to somehow transform the potential energy of interacting hadrons into their kinetic energy and into the masses of particles which appear due to resonance decays.

Here we would like to remind that traditionally the CFO is defined as the moment after which the inelastic reactions cease, while the evolution of hadronic matter is dominated by elastic re-scatterings towards kinetic freeze-out and decays of resonances [5,39]. Although it is an approximation, it provides a convenient and accurate framework to study the late stage of heavy ion collisions [5,39].

There are no a priori arguments to believe that the hardcore radius of all hadronic species should be the same, since the degree of cancellation of repulsive and attractive forces depends on the pair of interacting hadrons [31]. Therefore, a multicomponent HRGM (MHRGM), i.e. the HRGM with several different hard-core radii of hadrons, is required. The very high quality of the data description with $\chi^{2} /$ dof $\simeq 1.15$ [40-42] obtained by considering the individual hard-core radii $R_{\pi}$ for pions and $R_{K}$ for kaons, i.e. just two extra parameters, in addition to the traditional HRGM [39] which employs the hard-core radii $R_{b}$ of baryons and 
$R_{m}$ of other mesons, clearly demonstrates the advantages of the MHRGM.

Using the MHRGM it was possible for the first time to correctly reproduce the peaks of $K^{+} / \pi^{+}$and $\Lambda / \pi^{-}$ ratios without spoiling the high quality fit of other hadron yield ratios $[40,41]$. Then, by adding into the MHRGM the hard-core repulsion of (anti-) $\Lambda$ hyperons with their own hard-core radius it was possible to finally resolve the anti$\Lambda$ hyperon puzzle which in other HRGM like the one presented in [39] remained unsolved for years. Such an approach allowed us to get a very accurate description of all independent hadron multiplicity ratios measured in central nuclear collisions at the collision energies $\sqrt{s_{N N}}=2.7$, $3.3,3.8,4.3,4.9,6.3,7.7,8.8,9.2,12.3,17.3,62.4,130$, $200 \mathrm{GeV}$ with $\chi^{2} /$ dof $\simeq 0.96$ [43]. Despite this great success $[17-19,43]$ achieved in the solution [43] of the anti- $\Lambda$ puzzle [39], the present approach and the value $R_{\Lambda}=0.085$ fm for the hard-core radius of (anti-) $\Lambda$ hyperons [17-19] have not yet been used in other, far less realistic versions of the HRGM, and, moreover, this value was criticized on several occasions. Therefore, in this work we will explicitly demonstrate that the hyper-triton nuclear cluster and similar exotic light nuclei can provide us with an accurate tool to reliably determine the individual hard-core radii of short-lived hadrons like $\Lambda$ hyperons, if they form even a loosely bound state with nucleons.

It is apparent that the reliable analysis of light nuclear cluster multiplicities also requires an EoS with multicomponent hard-core repulsion [17]. However, until very recently there were two principal problems that did not allow one to develop the correct treatment of hard-core repulsion of light nuclear clusters. Firstly, the classical second virial coefficients of light nuclear clusters with hadrons were unknown and, hence, it was unclear how to correctly account for the hard-core repulsion among the light nuclei and hadrons in the MHRGM. Secondly, the Van der Waals treatment of multicomponent mixtures of gases is rather inconvenient, since for $N$ different hard-core radii of particles it is necessary to solve the system of (at least) $N$ transcendental equations and each of these equations contains a few hundreds of double integrals [40-43]. As a result, both a further development of the MHRGM based on Van der Waals approximation and its application to the cases $N \gg 1$ have no perspective.

Due to the fact that the classical second virial coefficients of light nuclear clusters were unknown, oversimplified and, hence, unrealistic assumptions about their interaction with hadrons were used. As a result such versions of the HRGM were unable to reproduce the available data with high accuracy and this fact stimulated several research groups of the heavy ion physics community to concentrate on the problem of light nuclear clusters and, in particular, on the PHTR. An almost complete list of the simpler versions of the HRGM can be found in $[44,45]$. Here we discuss the typical oversimpli- fying assumptions. Historically, the first version of HRGM that considered both hadrons and light nuclei clusters, treated them overly simplified as point-like particles [46]. In the second simplified HRGM description it was assumed that the hard-core radii of all light nuclear clusters are equal to the hard-core radius $R_{b}$ of baryons [47].

Surprisingly, these oversimplified HRGM of Refs. [46, 47] are able to describe the experimental data for hadronic and light nuclear clusters to some extent. A close inspection shows [17-19] that the reason for such an apparent success, which turns out to be a "pyrrhic victory", is that the local minimum of the light nuclear clusters $\chi_{A}^{2}$ as a function of their CFO temperature $T_{A}$, CFO baryonic chemical potential $\mu_{B}$ and CFO volume $V_{A}$ is very shallow and wide. Hence, the narrow $\chi_{h}^{2}$ minimum generated by the hadronic data is always dominant and, hence, one can poorly reproduce the total set of the hadronic and light nuclear clusters experimental data. Apparently, this is a self-cheating approach, since several erroneous assumptions can, in principle, produce the results which are not far from the truth on which, however, one cannot rely. Therefore, both from the academic and from the practical points of view it is absolutely necessary to develop a more realistic EoS to treat the light nuclear clusters.

Note that the existing shallow and wide minimum of $\chi_{A}^{2}$ of light nuclear clusters creates the problem to determine the most reliable CFO parameters of light nuclear clusters in the case, if their CFO occurs separately from that of the hadrons [19-21]. Such a problem cannot be resolved easily, even if one employs the most advanced MHRGM based on the IST EoS for the mixture of hadrons and light nuclear clusters $[18,19]$. Therefore, we were forced to search for an entirely new approach $[21,22]$ that is presented below.

\subsection{New strategy to analyze light nuclear cluster data}

In this work we are using the most advanced MHRGM with the IST EoS to fit the data measured by STAR and ALICE. This MHRGM was first heuristically obtained in [21], whereas in Ref. [22] it was rigorously derived from the grand canonical partition using the self-consistent method to treat the excluded volumes of multicomponent mixtures of classical [27] and quantum [26] particles with hard-core repulsion. In general the MHRGM with the IST EoS is a system of coupled equations for the pressure $p$ of considered system and its surface tension coefficient $\Sigma$ that is induced by the hard-core repulsion. This system can be written in the form

$$
\left\{\begin{aligned}
p & \equiv \sum_{k \in h, A} p_{k}=T \sum_{k \in h, A} \phi_{k} \exp \left[\frac{\mu_{k}-p V_{k}-\Sigma S_{k}}{T}\right], \\
\Sigma & \equiv \sum_{k \in h, A} \Sigma_{k}=\sum_{k \in h, A} R_{k} p_{k} \exp \left[-\frac{(\alpha-1) \Sigma S_{k}}{T}\right] \\
\mu_{k} & =\mu_{B} \mathcal{B}_{k}+\mu_{S} \mathcal{S}_{k}+\mu_{I_{3}} \mathcal{I}_{3 k} .
\end{aligned}\right.
$$


Here $p_{k}$ is the partial pressure of the k-th sort of particles, $\Sigma_{k}$ is their induced surface tension coefficient, $\mu_{B}, \mu_{S}$ and $\mu_{I 3}$ are, respectively, the baryonic, the strange and the third projection of isospin chemical potentials, while $\mathcal{B}_{k}, \mathcal{S}_{k}, \mathcal{I}_{3 k}$, respectively, denote the corresponding charges. $R_{k}, S_{k}$ and $V_{k}$ denote, respectively, the hard-core radius, the eigensurface and eigenvolume of the species $k$ of particles which are specified below. $\phi_{k}$ is the one-particle thermal partial density of the particle species $k$ with the Breit-Wigner mass distribution function

$$
\begin{aligned}
\phi_{k}= & g_{k} \gamma_{S}^{\left|s_{k}\right|} \int_{M_{k}^{T h}}^{\infty} \frac{d m}{N_{k}\left(M_{k}^{T h}\right)} \frac{\Gamma_{k}}{\left(m-m_{k}\right)^{2}+\Gamma_{k}^{2} / 4} \\
& \times \int \frac{d^{3} p}{(2 \pi \hbar)^{3}} \exp \left[-\frac{\sqrt{p^{2}+m^{2}}}{T}\right],
\end{aligned}
$$

where $g_{k}$ is the degeneracy factor of the k-sort of hadrons, $\gamma_{S}$ is the strangeness suppression factor [48], $\left|s_{k}\right|$ is the number of valence strange quarks and antiquarks in this kind of hadrons. The quantity $N_{k}\left(M_{k}^{T h}\right)$ denotes a corresponding normalization factor, while $M_{k}^{T h}$ corresponds to the decay threshold mass of the hadron $k$. For stable particles and for light nuclear clusters one should take the limit $\Gamma_{k} \rightarrow+0$ in Eq. (2).

The parameter $\alpha=1.25$ in Eq. (1) allows us to reproduce not only the second, but also the third and the fourth virial coefficients of classical hard spheres [18,19,24-26] and, hence, it enables us to go beyond the Van der Waals approximation. At the same time by choosing $\alpha=1$ one obtains a convenient system of equations for the Van der Waals EoS with the multicomponent hard-core repulsion [18, 19,24-26,34].

The summations in system (1) are made over all sorts of hadrons $h$ and nuclei $A \in\{1,2,3,4\}$ and their corresponding antiparticles which are considered as independent species. This system of equations is supplemented by the strange charge conservation law (see Ref. [18] for details).

From the system (1) one can determine the thermal particle number density of k-sort of particles [22] as

$\rho_{k} \equiv \frac{\partial p}{\partial \mu_{k}}=\frac{1}{T} \cdot \frac{p_{k} a_{22}-\Sigma_{k} a_{12}}{a_{11} a_{22}-a_{12} a_{21}}$,

where the coefficients $a_{i j}$ are defined as [22]

$$
\begin{aligned}
& a_{11}=1+\sum_{k \in h, A} V_{k} \frac{p_{k}}{T}, \\
& a_{12}=\sum_{k \in h, A} S_{k} \frac{p_{k}}{T}, \\
& a_{21}=\sum_{k \in h, A} V_{k} \frac{\Sigma_{k}}{T},
\end{aligned}
$$

$a_{22}=1+\alpha \sum_{k \in h, A} S_{k} \frac{\Sigma_{k}}{T}$.

To fit the experimental yields of hadrons, to the thermal particle number density (3) one has also to add the contributions coming from the decays of resonances. For the known branching ratios $B r_{l \rightarrow k}$ of hadronic decays $l \rightarrow k$ one can write the total yield of hadron $k$ as

$N_{k}^{\mathrm{tot}}=V\left[\rho_{k}+\sum_{l \neq k} \rho_{l} B r_{l \rightarrow k}\right]$,

with the CFO volume $V$. Then, the ratio $\mathcal{R}_{l k}=N_{k}^{\text {tot }} / N_{l}^{\text {tot }}$ of yields of the hadrons $l$ and $k$ can be found.

In the present work we are using a combined fit of particle yields and ratios, as dictated by the available data and by numerical convenience. Hence, the total $\chi_{\text {tot }}^{2}(V)$ is

$$
\begin{aligned}
\chi_{\text {tot }}^{2}(V)= & \chi_{R}^{2}+\chi_{Y}^{2}(V) \\
= & \sum_{k \neq l \in R}\left[\frac{\mathcal{R}_{k l}^{\text {theo }}-\mathcal{R}_{k l}^{\exp }}{\delta \mathcal{R}_{k l}^{\exp }}\right]^{2} \\
& +\sum_{k \in Y}\left[\frac{\rho_{k}(T) V-N_{k}^{\exp }}{\delta N_{k}^{\exp }}\right]^{2},
\end{aligned}
$$

where $\chi_{R}^{2}$ and $\chi_{Y}^{2}$ denote, respectively, the mean squared deviation for the ratios and for the yields.

All hard-core radii of hadrons, i.e. the hard-core radius of pions $R_{\pi}=0.15 \mathrm{fm}$, of kaons $R_{K}=0.395 \mathrm{fm}$, of (anti-) $\Lambda$ hyperons $R_{\Lambda}=0.085 \mathrm{fm}$, of other baryons $R_{b}=0.365 \mathrm{fm}$ and the one of other mesons $R_{m}=0.42 \mathrm{fm}$, are taken from $[18,19]$ (new radii in terms of Refs. $[18,19]$ ). These radii provide an excellent description of all independent hadron multiplicity ratios measured in central nuclear collisions at all AGS and all SPS energies, at the RHIC highest energies $\sqrt{s_{N N}}=62.4,130$ and $200 \mathrm{GeV}$ and at the ALICE energy $\sqrt{s_{N N}}=2.76 \mathrm{TeV}$ with $\chi^{2} /$ dof $\simeq 1.13$ [19]. Then the eigensurface and eigenvolume of the $k$-th sort of hadrons are defined as $S_{k}=4 \pi R_{k}^{2}$ and $V_{k}=\frac{4}{3} \pi R_{k}^{3}$, respectively.

To take into account the classical excluded volumes of light nuclear clusters and hadrons we employ two approaches worked out in [20-22] with one exception, namely here we consider the hyper-triton (HTR) differently compared to our previous studies in Refs. [20-22]. Both of these approaches use the classical second virial coefficient or in other words excluded volumes per particle of light nuclear clusters of $A \in\{1,2,3,4\}$ baryonic constituents and hadron $h[21,22]$

$b_{A h}=b_{h A}=A \frac{2}{3} \pi\left(R_{b}+R_{h}\right)^{3}$,

which can be easily found from the fact that all light nuclear clusters analyzed here are roomy clusters. From the fact that in classical mechanics the light nuclear clusters of $A$ nucle- 
ons are roomy clusters, i.e. the mean classical distance among each nucleon inside of such clusters is very large compared to hard-core radii of hadrons (for deuterons of mean radius $R_{r m s}$, for example, the mean distance is $2 R_{r m s} \simeq 4.28 \mathrm{fm}$, see Ref. [22] for more details), one can immediately deduce that it is possible to freely translate the hadron $h$ with the hardcore radius $R_{h}$ around each constituent of a nucleus without touching any other constituent of this nucleus. Then the excluded volume, or volume (per particle) which is unavailable for the hadron of hard-core radius $R_{h}$, originated from the translation of this hadron around each of $A$ nucleons inside the considered nucleus of hard-core radius $R_{b}$ is given by Eq. (10).

The main approximation used to obtain Eq. (10) is that we consider the nucleus as a classical composite object. Apparently, if one of the nucleons inside the nucleus is replaced by the $\Lambda$-hyperon with the hard-core radius $R_{\Lambda}$, the excluded volume formula (10) should be modified to

$$
\begin{aligned}
{ }^{\Lambda} b_{A h} & ={ }^{\Lambda} b_{h A} \\
& =(A-1) \frac{2}{3} \pi\left(R_{b}+R_{h}\right)^{3}+\frac{2}{3} \pi\left(R_{\Lambda}+R_{h}\right)^{3} .
\end{aligned}
$$

Here we use two approaches to calculate the thermal density of particles of sort $k$ using expression (3), which can be found from the solution of the system (1), supplemented by $R_{k}, S_{k}$ and $V_{k}$ and the excluded volumes of (10) and (11) (see later). The first of these approaches, named as the IST EoS, is rigorously derived using a self-consistent treatment of classical excluded volumes of light (anti-)nuclei and hadrons [22]. In this approach the parameters $R_{A}, S_{A}$ and $V_{A}$ of light nuclear clusters with the mass number $A$ entering the system (1) are given as functions of the baryon radius by

$R_{A}=A \cdot R_{b}, S_{A}=A \cdot 4 \pi R_{b}^{2}, V_{A}=A \cdot \frac{4}{3} \pi R_{b}^{3}$

The second approach is approximate and complementary to the first one. It is based on an approximate, but rather accurate treatment of the equivalent hard-core radius of roomy nuclear cluster and pions which are the dominating component of the HRG at the energy range of our interest. In this approach one can find an effective hard-core radius of nuclei as $R_{A} \simeq A^{1 / 3} R_{b}$, since the hard-core radius of pions is very small and it generates a negligible correction to $R_{A}$ [22]. Consequently, the eigensurface $S_{A}$ and eigenvolume $V_{A}$ of light nuclear clusters to be used in the system (1) can be written as

$$
R_{A} \simeq A^{\frac{1}{3}} R_{b}, \Rightarrow S_{A} \simeq A^{\frac{2}{3}} \cdot 4 \pi R_{b}^{2}, \quad V_{A} \simeq A \cdot \frac{4}{3} \pi R_{b}^{3}
$$

The hard-core radius of light nuclear clusters defined in this way is similar to the expression of the Bag Model [49]. Hereafter this model is called the BMR EoS.
Applying the Eqs. (12) and (13) to the PHTR ${ }_{\Lambda}^{3} \bar{H} /{ }^{3} \overline{\mathrm{He}}$ and ${ }_{\Lambda}^{3} H /{ }^{3} \mathrm{He}$ measured by STAR at $\sqrt{s_{N N}}=200 \mathrm{GeV}[11,12]$, in Ref. [22] we obtained a very good overall fit of seven data points for the CFO temperature of nuclei $T_{A}=167.3 \pm 3.93$ $\mathrm{MeV}$ with $\chi_{A}^{2} \simeq 9.6$ and for $T_{A}=240.3 \pm 21.38 \mathrm{MeV}$ with $\chi_{A}^{2} \simeq 4.92$ (see Fig. 4 and Table 3 in [22]). Nevertheless, to our surprise, even at the high temperature $T_{A}=240.3 \mathrm{MeV}$ the mean squared deviation of these PHTR alone is large and it defines the whole value of $\chi_{A}^{2} \simeq 4$.92. In other words, even these advanced approaches are not doing a better job for the PHTR than the other thermal models cited in [44,45].

A close inspection of Eqs. (12) and (13) enabled us to find an elegant solution to this puzzle, which, nevertheless, requires the most natural generalization of the two approaches mentioned above to the case of nuclei with $A$ constituents which can be divided into $N_{S}$ different sorts

$A=\sum_{k=1}^{N_{s}} n_{k}$, with $n_{k} \geq 1$,

where $n_{k} \in Z$ is the number of constituents of the $k$-th sort inside the nuclei.

For the IST EoS approach the generalization (14) leads to a set of simple replacements

$R_{A} \rightarrow \sum_{k=1}^{N_{s}} n_{k} R_{k}, S_{A} \rightarrow \sum_{k=1}^{N_{s}} n_{k} S_{k}, V_{A} \rightarrow \sum_{k=1}^{N_{s}} n_{k} V_{k}$,

where the sums are running over the constituent hadrons of sort $k$ having the hard-core radius $R_{k}$, the eigensurface $S_{k}$ and eigenvolume $V_{k}$. This is a generalization of Eq. (11). For the HTR one can write the Eq. (15) explicitly as

$$
\begin{aligned}
R_{H T R} & =2 \cdot R_{b}+R_{\Lambda}, \\
S_{H T R} & =2 \cdot 4 \pi R_{b}^{2}+4 \pi R_{\Lambda}^{2}, \\
V_{H T R} & =2 \cdot \frac{4}{3} \pi R_{b}^{3}+\frac{4}{3} \pi R_{\Lambda}^{3} .
\end{aligned}
$$

The system (1), supplemented by the quantities $R_{H T R}, S_{H T R}$ and $V_{H T R}$, is called hereafter the IST $\Lambda$ EoS.

In order to modify the BMR EoS one should introduce the equivalent hard-core radius $R_{A h}$ for a pair of a nucleus with $A$ constituents and an effective hadron $h$ with the mean hard-core radius $\bar{R}$ defined later. Then equating the excluded volume $\frac{2}{3} \pi\left(R_{A h}\right)^{3}$ to the second virial coefficient of such a pair $b_{A h}$ of Eq. (10), one finds

$b_{A h}=\frac{2}{3} \pi\left(R_{A}+\bar{R}\right)^{3}=\frac{2}{3} \pi\left[\sum_{k=1}^{N_{s}} n_{k}\left(R_{k}+\bar{R}\right)^{3}\right]$,

or explicitly

$R_{A h}=R_{A}+\bar{R}=\left[\sum_{k=1}^{N_{s}} n_{k}\left(R_{k}+\bar{R}\right)^{3}\right]^{\frac{1}{3}}$ 
Now from the expression for $R_{A h}$ one can determine the effective hard-core radius of a nucleus in a hadronic medium with the mean hard-core radius $\bar{R}$

$R_{A}=R_{A h}-\bar{R}=\left[\sum_{k=1}^{N_{s}} n_{k}\left(R_{k}+\bar{R}\right)^{3}\right]^{\frac{1}{3}}-\bar{R}$.

Applying Eq. (17) to the HTR in a medium dominated by pions, one obtains

$R_{H T R}=\left[2\left(R_{b}+R_{\pi}\right)^{3}+\left(R_{\Lambda}+R_{\pi}\right)^{3}\right]^{\frac{1}{3}}-R_{\pi}$.

Hereafter this prescription for the hard-core radius of light nuclear clusters is called as the BMR $\Lambda$ EoS.

Note that due to the inequality $R_{\Lambda} \ll R_{b}$ the terms with $R_{\Lambda}$ are almost negligible and, hence, the excluded volume of HTR is practically the same as the deuteron one.

If the medium has different properties, for instance, if it is baryon dominated, the mean hard-core radius of the hadronic mixture can be found from the solution of system (1) as

$\bar{R}=\frac{\Sigma}{p} \simeq \frac{\sum_{k \in h} \quad R_{k} p_{k} \exp \left[-\frac{(\alpha-1) \Sigma S_{k}}{T}\right]}{\sum_{k \in h} p_{k}}$,

where the contribution of nuclei in the sums above is neglected. Note that the quantity $\bar{R}$, indeed, is defined in this way in the self-consistent treatment of second virial coefficients of mixture of hadrons and nuclei developed recently in [22]. Eq. (21) shows that the main contribution to the mean radius $\bar{R}$ is generated by the particles with highest value of partial pressure. Apparently, these are the lightest particles with the smallest hard-core radius. For high temperatures and low baryonic charge densities these are pions, while for baryon rich matter it should be found from Eq. (21) using the solution of system (1). From Eq. (21) one can see that for very high particle number densities (typically above the CFO ones) the mean radius $\bar{R}$ gets smaller due to the suppression factors $\exp \left[-\frac{(\alpha-1) \Sigma S_{k}}{T}\right]<1$ which are generated by the induced surface tension coefficient for $\alpha>1$.

\section{Analysis of light nuclei multiplicities measured in A + A collisions}

Following our original idea [20], in this section we consider not only the traditional scenario of a simultaneous CFO of hadrons and light nuclear clusters (single CFO), but also the scenario of their separate freeze-outs (separate CFO). In contrast to hadrons which are tightly bound objects the light nuclei are loosely bound and, therefore, it is hard to believe that their evolution is similar to the one of hadrons. Consequently, there is no a priori reason to assume that the CFO of light nuclear clusters coincides with the one of hadrons.
Nevertheless, the success of the MHRGM in describing the multiplicities of hadrons and light nuclear clusters provides an evidence that their thermalization mechanisms may be similar. In our previous works [20-22] we argued that the light nuclear clusters are produced at the moment of hadronization of quark-gluon bags. Since the quark-gluon bags with the Hagedorn mass spectrum [50] are perfect thermostats and perfect particle reservoirs [51], any particle or cluster emitted by such a bag will be produced in a full chemical and thermal equilibrium with it [51]. In a recent preprint [62] based on a transport model which takes into account the microscopic reactions between hadrons and heavy resonances with the Hagedorn mass spectrum it is shown that such an approach is able to reasonably well reproduce the ALICE data on hadronic and light nuclei multiplicities. In principle, such a hypothesis is not only able to explain the fact that the light nuclear clusters appear in full chemical and thermal equilibrium, but also the scale of their CFO temperatures extracted from the ALICE and STAR data in [22]. Of course, the corresponding model is highly required, but before its development one has to reliably extract the parameters of the CFO of light nuclear clusters.

It is also necessary to remind that the hypothesis of simultaneous chemical and kinetic freeze-out of heavy particles [52-55] which do not form resonances with pions was suggested a long time ago. It was argued [52-55] that such a simultaneous freeze-out occurs at the hadronization of quarkgluon bags. The validity of such a hypothesis was for the first time demonstrated for $\Omega$ hyperons and $J / \psi$ and $\psi^{\prime}$ mesons in Refs. [52,53] and recently it was extended [22] to the CFO of light nuclear clusters in high energy nuclear collisions.

Besides, the hypothesis of separate $\mathrm{CFO}$ was proved to be very successful in explaining the nuances of the CFO of strange hadrons $[42,56]$. The strangeness suppression factor $\gamma_{S}$ was introduced in Ref. [48] to quantify the deviation of strange charge from chemical equilibrium. However, as it was for the first time shown in $[42,56]$ such a factor is unnecessary to describe the hadronic multiplicities, since the data can be perfectly explained by the fact that the CFO of strange hadrons occurs on a separate hyper-surface compared to the one for hadrons which consist of the $u$ and $d$ (anti)quarks $[42,56]$. More sophisticated scenarios of the CFO of strange hadrons were suggested in Refs. [57,58].

The new strategy based on a combined description of the same set of data achieved independently by the IST and BMR EoS worked quite well [22] with the inaccurate prescription of Eq. (12) for the HTR excluded volume and the one of Eq. (13) for the effective hard-core radius of HTR. It allowed us to easily distinguish the scenarios of single and separate $\mathrm{CFO}$ of light nuclear clusters. Therefore, it is a very intriguing question what this strategy will give us for the correct prescriptions (16) and (20) worked out here. 

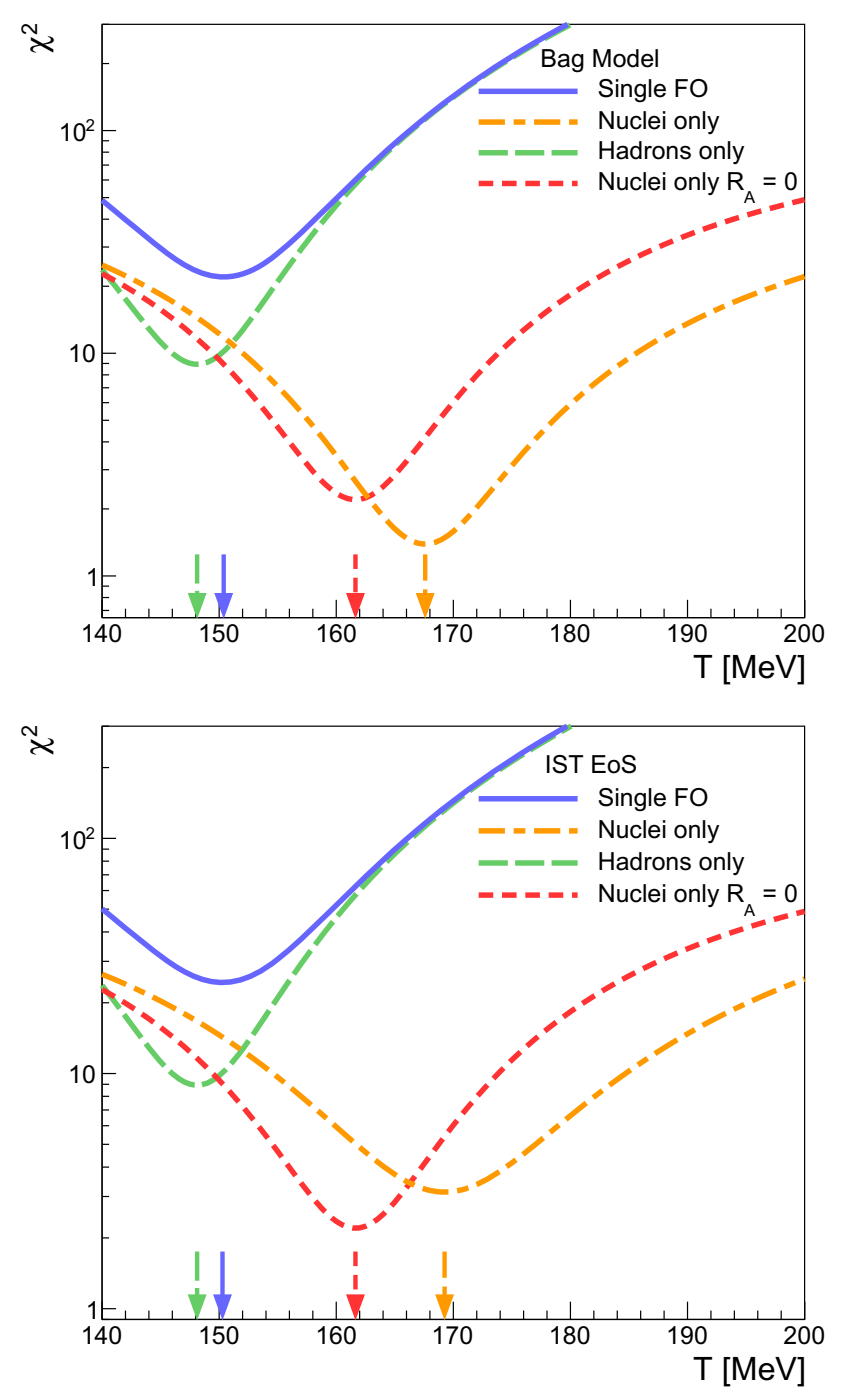

Fig. 1 Temperature dependence of $\chi_{\text {tot }}^{2}, \chi_{h}^{2}$ and $\chi_{A}^{2}$ for fit of ALICE data measured at $\sqrt{s_{N N}}=2.76 \mathrm{TeV}$. Upper panel: The dependence obtained with the BMR $\Lambda$ EoS. Lower panel: The dependence obtained with the IST $\Lambda$ EoS

However, before going into a discussion of numerics we have to remind that in the present work we follow the setup of Refs. [19,22], i.e. the hard-core radii of hadrons (new radii of Refs. [19]) given above, the particle table along with their decays and all the hadronic data of the STAR Collaboration measured at $\sqrt{s_{N N}}=200 \mathrm{GeV}$ and the ones of the ALICE Collaboration measured at $\sqrt{s_{N N}}=2.76 \mathrm{TeV}$ are taken from Refs. [19,22]. Since all these elements of the MHRGM are well documented, they can be found in the original works $[19,22]$, while here we concentrate on the new and essential features of the novel MHRGM worked out here.

First we analyze the ALICE Collaboration data measured at $\sqrt{s_{N N}}=2.76 \mathrm{TeV}$. From Fig. 1 and Table 1 one can see that the results on $\chi^{2}$ obtained with the BMR $\Lambda$ and IST $\Lambda$ EoS are close to the ones which were obtained in [22]. An apparent reason for such a similarity is that all generic features of the present results coincide with the ones found in [22].

In the single CFO scenario we have 10 experimental data points for hadronic ratios, 8 yields of light nuclear clusters and 2 fitting parameters (CFO temperatures $T_{h}=T_{A}$ and volume $V$ ), while all chemical potentials are set to zero $[19,22]$. Similarly to the results of Ref. [22] we see that the CFO temperatures $T_{h}$ found by the BMR $\Lambda$ and IST $\Lambda$ EoS for the single CFO scenario are practically the same and, moreover, they coincide with the ones found in [22]. Indeed, from Table 1 one can see that for this scenario the CFO temperatures $\left.T_{h}\right|_{B M R \Lambda}=150.29 \pm 1.92 \mathrm{MeV}$ and $\left.T_{h}\right|_{I S T \Lambda}=150.39 \pm 1.90 \mathrm{MeV}$ differ from each other on $0.1 \mathrm{MeV}$ which is negligible difference compared to their errors. This is not surprising, since for the single CFO scenario the minimum of $\chi_{t o t}^{2} / d o f$ is mainly defined by the hadronic yields.

For the scenario of separate CFO of hadrons and light nuclear clusters one obtains a very similar behavior of $T_{h}$ and $T_{A}$ found by the BMR $\Lambda$ and IST $\Lambda$ EoS, although the number of fitting parameters is 3 , since $T_{h} \neq T_{A}$. Therefore, in contrast to our previous findings, the refined strategy based on Eqs. (16) and (20) automatically provides a very close location of the minima of light nuclear clusters $\chi_{A}^{2}$ obtained by the BMR $\Lambda$ and IST $\Lambda$ EoS. Consequently, with the refined strategy one does not need to search for the common description of the data by two models, since such a description is automatically located within the error bars of the found CFO parameters. Nevertheless, the BMR $\Lambda$ and IST $\Lambda$ EoS provide an additional cross-check of the obtained results.

Moreover, the main conclusion of Ref. [22] that at ALICE energy of collisions the separate CFO of light nuclei is the most probable one, is confirmed by the more accurate MHRGM worked out here with high accuracies $\chi_{\text {tot }}^{2} /\left.d o f\right|_{I S T \Lambda}=0.753$ and $\chi_{\text {tot }}^{2} /\left.d o f\right|_{B M R \Lambda}=0.676$. The main difference with Ref. [22] is that the CFO temperature of light nuclear clusters $T_{A}^{c o m}=175.1_{-3.9}^{+2.3} \mathrm{MeV}$ [22] decreased now by a few $\mathrm{MeV}$ to the value $\left.T_{A}\right|_{I S T \Lambda}=169.25 \pm 5.57$ $\mathrm{MeV}$ as one can see from Table 1 for the separate CFO scenario. Apparently, these values of the CFO temperature of light nuclear clusters overlap and this means that there is no any inconsistency in the obtained results here and in Ref. [22] and between the employed versions of EoS.

The results of the ALICE data fits are shown in Fig. 2 for the scenario of separate CFO. From this figure one can see that all experimental yields of light nuclear clusters are described with deviations within $\simeq 1 \sigma$. Since the quality of the hadronic data description is the same as in [22]), these figures are not shown here.

More interesting results are obtained for the light nuclear clusters data measured by the STAR Collaboration at $\sqrt{s_{N N}}=200 \mathrm{GeV}$. These STAR data consist of 10 hadronic ratios (for more details see [22]), the (anti-)deuteron yields 
Table 1 Results for the fit of ALICE data measured at $\sqrt{s_{N N}}=2.76 \mathrm{TeV}$ obtained by the advanced HRGM. The CFO temperature of hadrons is $T_{h}$, the CFO temperature of light (anti)nuclei is $T_{A}$, while their CFO volume is $V_{A}$. The last column gives the fit quality

\begin{tabular}{llll}
\hline Description & $T_{h}, \mathrm{MeV}$ & $T_{A}, \mathrm{MeV}$ & $V_{A}, \mathrm{fm}^{3}$ \\
\hline Single CFO, IST $\Lambda$ & $150.29 \pm 1.92$ & $150.29 \pm 1.92$ & $13145 \pm 2233$ \\
Single CFO, BMR $\Lambda$ & $150.39 \pm 1.90$ & $150.39 \pm 1.90$ & $11201 \pm 2009$ \\
Separate CFO, IST $\Lambda$ & $148.12 \pm 2.03$ & $169.25 \pm 5.57$ & $3898 \pm 1272$ \\
Separate CFO, BMR $\Lambda$ & $148.12 \pm 2.03$ & $167.59 \pm 5.39$ & $3123 \pm 1198$ \\
\hline
\end{tabular}
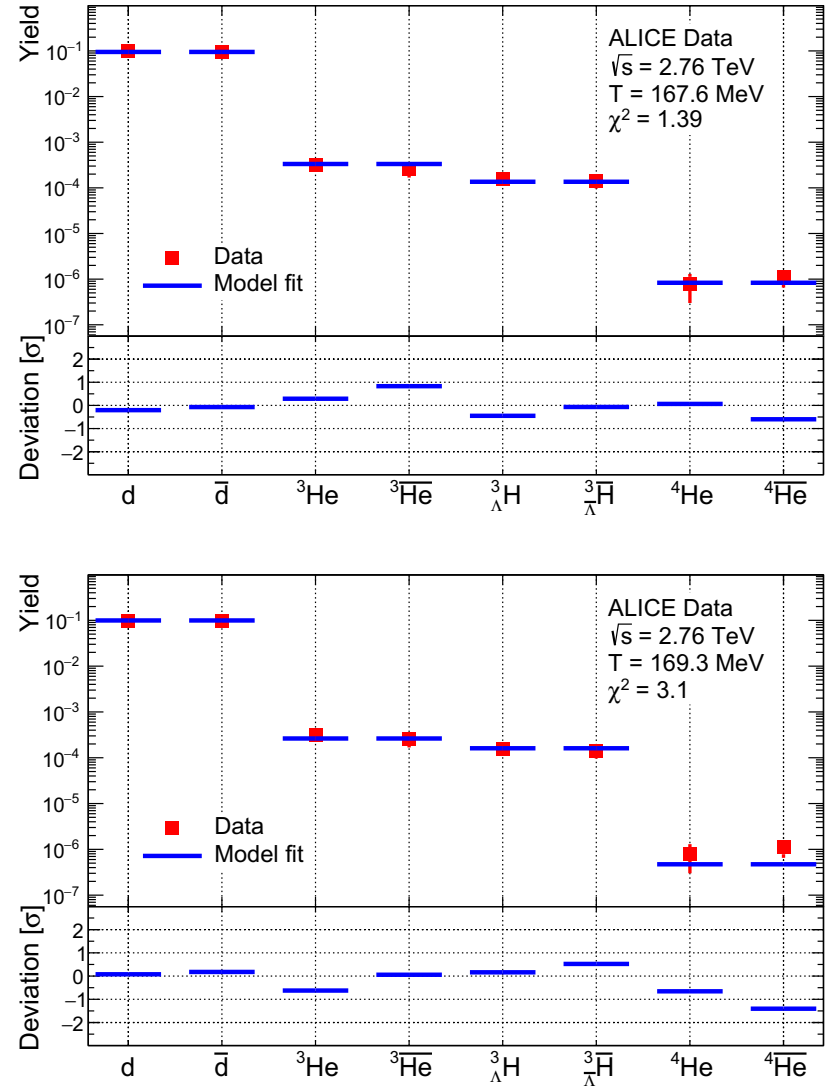

Fig. 2 The yields of nuclear clusters measured at $\sqrt{s_{N N}}=2.76 \mathrm{TeV}$ by ALICE vs. theoretical description in the scenario of separate CFO of light (anti)nuclei. Insertion shows the deviation of theory from data in the units of experimental error. Upper panel: The $\min \chi_{A}^{2}(V)$ corresponds to the BMR $\Lambda$ EoS. Lower panel: Same as in the upper panel, but for the IST $\Lambda$ EoS

[13] and 5 other light nuclear clusters ratios [11,12]. They contain the PHTR ratios which no one could describe for almost a decade. From Table 2 one can see that the results obtained by the IST $\Lambda$ EoS for both CFO scenarios are practically the same and their fit quality is $\chi_{\text {tot }}^{2} / d o f \simeq 1$, i.e. it is essentially reduced compared to Ref. [22].

From Fig. 3 one can see that for the separate CFO of light nuclear clusters both EoS provide the CFO temperature of nuclei $T_{A}$ above $180 \mathrm{MeV}$ (in this case there are 5 fitting parameters such as the CFO temperature of hadrons (nuclei)
$T_{h}\left(T_{A}\right)$, the CFO baryonic chemical potential of hadrons (nuclei) $\mu_{B}^{h}\left(\mu_{B}^{A}\right)$ and the CFO volume of nuclei $\left.V_{A}\right)$. Note, however, that according to lattice version of QCD at vanishing value of the baryonic chemical potential $[35,36]$ it is rather problematic to use the hadronic EoS for these CFO temperatures, since this is the region above the cross-over to quark-gluon plasma. Although for the separate CFO scenario all the PHTR are reproduced by the IST $\Lambda$ EoS with the deviation smaller than $1 \sigma$ (see Fig. 4), this scenario can be ruled out by requiring a consistency with the lattice QCD results.

As an independent indicator in favor of the single CFO scenario for the STAR energies the $S_{3}$ and $\bar{S}_{3}$ ratios

$S_{3}=\frac{{ }_{\Lambda}^{3} \mathrm{H}}{{ }^{3} \mathrm{He} \times \frac{\Lambda}{p}}, \quad \bar{S}_{3}=\frac{\frac{3}{\Lambda} \overline{\mathrm{H}}}{{ }^{3} \overline{\mathrm{He}} \times \frac{\bar{\Lambda}}{\bar{p}}}$,

can be used. From Fig. 5 one can clearly see that these special ratios provided by the STAR Collaboration [11] are well reproduced for the single CFO scenario found by the IST $\Lambda$ EoS. In our opinion it is absolutely remarkable that the data on the $S_{3}$ and $\bar{S}_{3}$ ratios, which were not used in our fits, are reproduced by the most elaborate version of the MHRGM for the single CFO scenario. In this case there are only 3 fitting parameters, namely the $\mathrm{CFO}$ temperature of hadrons and nuclei $T_{h}=T_{A}$, the CFO baryonic chemical potential of hadrons and nuclei $\mu_{B}^{h}=\mu_{B}^{A}$ and the CFO volume of nuclei $V_{A}$.

Using the CFO parameters determined from the fits of STAR and ALICE data discussed above, we made predictions for the yields of some light nuclear clusters which are also discussed in [59] (see Tables 3 and 4) with different CFO scenarios. As one can see from Tables 3 and 4 the absolute yields of the light nuclear clusters are somewhat higher than those of the separate CFO scenario and, therefore, the new measurements of some of these nuclei can help to distinguish the scenarios of $\mathrm{CFO}$ of nuclei. Furthermore, comparison of our predictions with the ones obtained for the same collision energies by the advanced coalescence model [60] (see Table II therein) shows that our numbers are systematically higher. Therefore, the new measurements of the yields of such nuclei as ${ }_{\Lambda}^{4} \mathrm{H},{ }_{\Lambda}^{4} \mathrm{He}$ and ${ }_{\Lambda \Lambda}^{4} \mathrm{H}$ maybe of crucial importance for reli- 
Table 2 Results for the fit of STAR data measured at $\sqrt{s_{N N}}=200 \mathrm{GeV}$ obtained by the advanced HRGM. The CFO temperature of hadrons (nuclei) is $T_{h}\left(T_{A}\right)$, the CFO baryonic chemical potential of hadrons (nuclei) is $\mu_{B}^{h}\left(\mu_{B}^{A}\right)$, while the CFO volume of nuclei is $V_{A}$. The last column gives the fit quality

\begin{tabular}{|c|c|c|c|c|c|c|}
\hline Description & $T_{h}, \mathrm{MeV}$ & $T_{A}, \mathrm{MeV}$ & $\mu_{B}^{h}, \mathrm{MeV}$ & $\mu_{B}^{A}, \mathrm{MeV}$ & $V_{A}, \mathrm{fm}^{3}$ & $\chi^{2} / d o f$ \\
\hline Single CFO, IST $\Lambda$ & $168.30 \pm 3.85$ & $168.30 \pm 3.85$ & $30.12 \pm 3.27$ & $30.12 \pm 3.27$ & $2056 \pm 375$ & 1.069 \\
\hline Single CFO, BMR $\Lambda$ & $167.43 \pm 3.84$ & $167.43 \pm 3.84$ & $30.00 \pm 3.26$ & $30.00 \pm 3.26$ & $1667 \pm 355$ & 1.339 \\
\hline Separate CFO, IST $\Lambda$ & $166.51 \pm 4.07$ & $185.99 \pm 9.09$ & $28.84 \pm 5.37$ & $34.30 \pm 4.81$ & $1093 \pm 278$ & 0.995 \\
\hline Separate CFO, BMR $\Lambda$ & $166.51 \pm 4.07$ & $182.69 \pm 14.1$ & $28.84 \pm 5.37$ & $33.30 \pm 4.94$ & $831 \pm 455$ & 1.459 \\
\hline
\end{tabular}
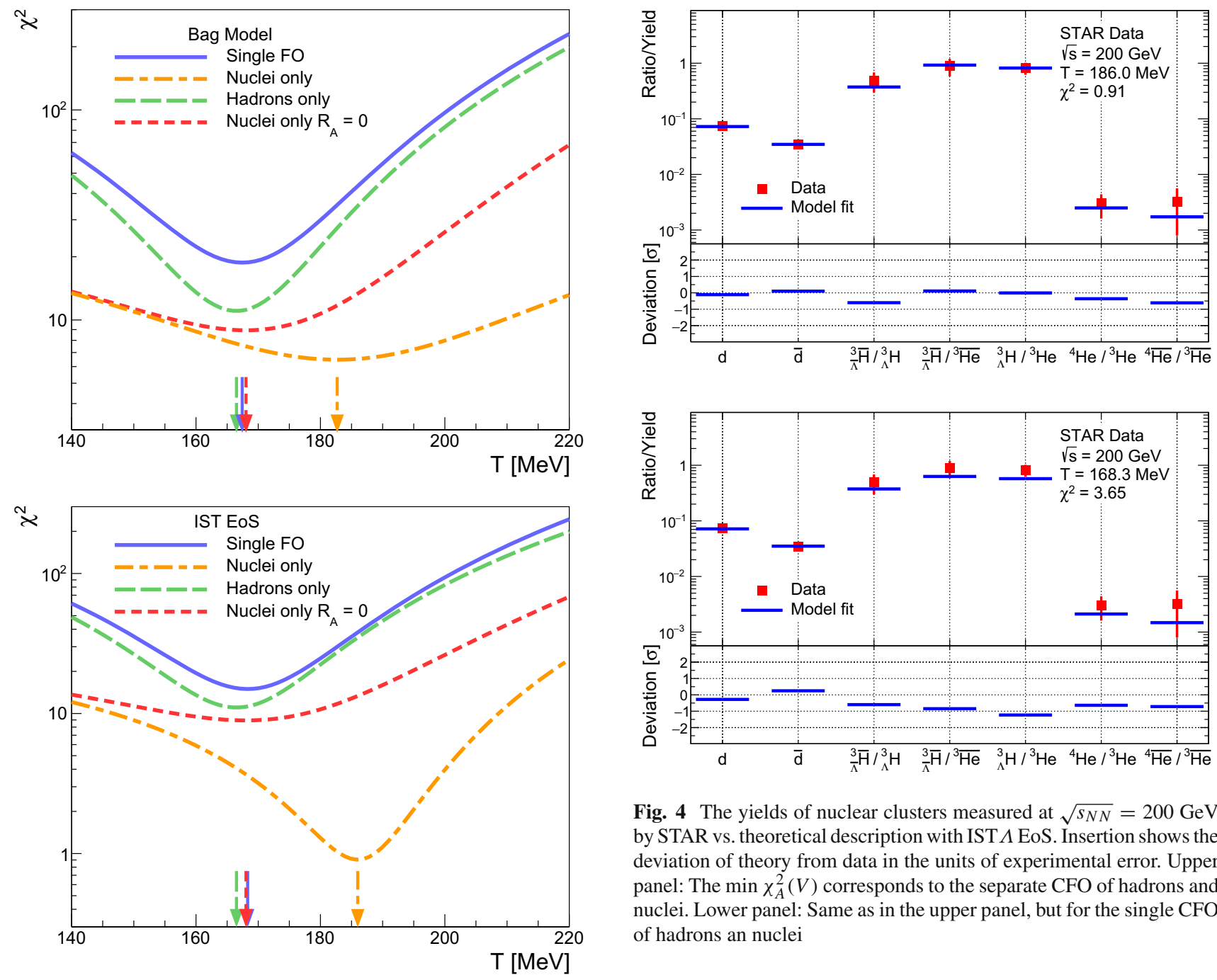

Fig. 4 The yields of nuclear clusters measured at $\sqrt{s_{N N}}=200 \mathrm{GeV}$ by STAR vs. theoretical description with IST $\Lambda$ EoS. Insertion shows the deviation of theory from data in the units of experimental error. Upper panel: The $\min \chi_{A}^{2}(V)$ corresponds to the separate CFO of hadrons and nuclei. Lower panel: Same as in the upper panel, but for the single CFO of hadrons an nuclei

Fig. 3 Temperature dependence of $\chi_{\text {tot }}^{2}, \chi_{h}^{2}$ and $\chi_{A}^{2}$ for fit of STAR data measured at $\sqrt{s_{N N}}=200 \mathrm{GeV}$. Upper panel: The dependence obtained with the BMR $\Lambda$ EoS. Lower panel: The dependence obtained with the IST $\Lambda$ EoS

able determination of the CFO scenarios in the MHRGM and in the coalescence model.

\section{Conclusions and perspectives}

In this work we obtained an accurate description of the hadronic and light nuclear clusters data measured by the STAR Collaboration at $\sqrt{s_{N N}}=200 \mathrm{GeV}$ and by the ALICE LHC at $\sqrt{s_{N N}}=2.76 \mathrm{TeV}$ with $\chi^{2} / d o f \simeq \frac{26.261}{18-3+17-3} \simeq$ 0.91 for two best fits of combined data. This is achieved by applying the new strategy of analyzing the light nuclear clusters data and by using the correct value of the hard-core radius of the (anti-) $\Lambda$ hyperons in the expressions for the clas- 

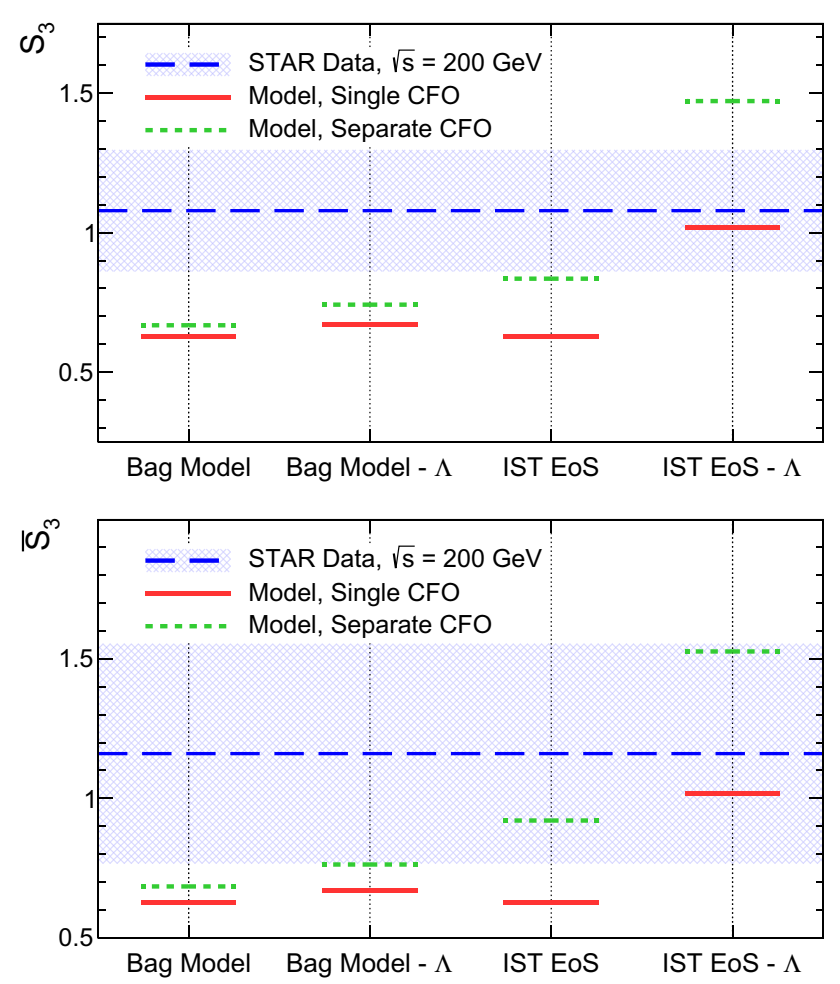

Fig. 5 Upper panel: $S_{3}$ ratio measured at $\sqrt{s_{N N}}=200 \mathrm{GeV}$ by STAR [11] vs. theoretical description obtained for different CFO scenarios and EoS. Lower panel: Same as in the upper panel, but for $\bar{S}_{3}$ ratio [11]

sical second virial coefficients and for the equivalent hardcore radius of HTR. The generalized expressions for these quantities are worked out here. Using them we obtained a greatly improved description of the STAR data compared to our recent analysis done in [22]. At the same time for the ALICE data we obtained almost the same results and the same high quality of the fit.
The fact, that the hard-core radius of the (anti-) $\Lambda$ hyperons $R_{\Lambda}$ is essentially smaller than the one of other hadrons was for the first time justified in Ref. [43]. The high quality description of the hadronic multiplicities measured at the collision energies $\sqrt{s_{N N}}=2.7,3.3,3.8,4.3,4.9$, $6.3,7.7,8.8,9.2,12.3,17.3,62.4,130,200,2760 \mathrm{GeV}$ obtained by the most elaborate version of MHRGM with the IST EoS for the same set of hadronic hard-core radii $[18,19]$ confirmed this conclusion about the hard-core radius of the (anti-) $\Lambda$ hyperons. Finally, the resolution of the PHTR puzzle provides a strong support for the model assumption that $R_{\Lambda} \simeq 0.085 \mathrm{fm}$ is much smaller than $R_{b} \simeq 0.365 \mathrm{fm}$. This is in accord with the expectation from Pauli blocking phenomenology, because nucleons and hyperons have a different quark content so that the repulsive force induced by quark exchange effects between them is much smaller than between nucleons.

One of the most striking findings of this work is that for the most probable scenario of CFO for the STAR energy, i.e. for a single $\mathrm{CFO}$ of hadrons and nuclei, the obtained parameters allowed us to predict the ratios $S_{3}$ and $\bar{S}_{3}$ [11] in accordance with the experimental data without fitting them at all. Apparently, this fact evidences about an internal consistency of the approach based on the IST $\Lambda$ EoS and about a high reliability of the obtained results. Based on these findings we predicted the absolute yields of several light nuclear clusters including some exotic ones for the considered energies of collisions.

In contrast to all previous findings, the CFO temperature $T_{A} \simeq 168.75 \pm 5.57 \mathrm{MeV}$ of the most probable scenarios of light nuclear clusters CFO is found to be the same for the STAR and ALICE data. Above it was argued that this is the hadronization temperature of quark-gluon bags. Note that this conclusion is in line with the lattice QCD results for the chiral cross-over transition region which at vanishing

Table 3 Expected yields of some (hyper-)nuclei per event obtained by the advanced HRGM for the fit of STAR data measured at $\sqrt{s_{N N}}=200$ $\mathrm{GeV}$ with different $\mathrm{CFO}$ scenarios and nuclear cluster radii estimations. The CFO scenario is specified in parentheses

\begin{tabular}{lllllllll}
\hline Particle & $\begin{array}{l}\text { BMR EoS } \\
(\text { Single })\end{array}$ & $\begin{array}{l}\text { IST EoS } \\
(\text { Single })\end{array}$ & $\begin{array}{l}\text { BMR } \Lambda \text { EoS } \\
(\text { Single })\end{array}$ & $\begin{array}{l}\text { IST } \Lambda \text { EoS } \\
(\text { Single })\end{array}$ & $\begin{array}{l}\text { BMR EoS } \\
(\text { Separate) }\end{array}$ & $\begin{array}{l}\text { IST EoS } \\
(\text { Separate) }\end{array}$ & $\begin{array}{l}\text { BMR } \Lambda \text { EoS } \\
(\text { Separate })\end{array}$ & $\begin{array}{l}\text { IST } \Lambda \text { EoS } \\
(\text { Separate })\end{array}$ \\
\hline${ }^{3} \mathrm{H}$ & $3.0 \times 10^{-4}$ & $2.2 \times 10^{-4}$ & $3.0 \times 10^{-4}$ & $2.2 \times 10^{-4}$ & $4.0 \times 10^{-4}$ & $3.5 \times 10^{-4}$ & $4.2 \times 10^{-4}$ & $2.8 \times 10^{-4}$ \\
${ }^{3} \mathrm{He}$ & $3.0 \times 10^{-4}$ & $2.2 \times 10^{-4}$ & $3.0 \times 10^{-4}$ & $2.2 \times 10^{-4}$ & $4.0 \times 10^{-4}$ & $3.5 \times 10^{-4}$ & $4.2 \times 10^{-4}$ & $2.8 \times 10^{-4}$ \\
${ }^{4} \mathrm{He}$ & $8.8 \times 10^{-7}$ & $4.6 \times 10^{-7}$ & $8.9 \times 10^{-7}$ & $4.7 \times 10^{-7}$ & $1.6 \times 10^{-6}$ & $1.3 \times 10^{-6}$ & $1.7 \times 10^{-6}$ & $7.0 \times 10^{-7}$ \\
${ }_{\Lambda}^{3} \mathrm{H}$ & $1.0 \times 10^{-4}$ & $7.7 \times 10^{-5}$ & $1.1 \times 10^{-4}$ & $1.3 \times 10^{-4}$ & $1.5 \times 10^{-4}$ & $1.6 \times 10^{-4}$ & $1.7 \times 10^{-4}$ & $2.3 \times 10^{-4}$ \\
${ }_{\Lambda}^{4} \mathrm{H}$ & $1.1 \times 10^{-6}$ & $5.9 \times 10^{-7}$ & $1.0 \times 10^{-6}$ & $1.0 \times 10^{-6}$ & $2.1 \times 10^{-6}$ & $2.3 \times 10^{-6}$ & $2.1 \times 10^{-6}$ & $2.2 \times 10^{-6}$ \\
${ }_{\Lambda}^{4} \mathrm{He}$ & $1.1 \times 10^{-6}$ & $5.9 \times 10^{-7}$ & $1.0 \times 10^{-6}$ & $1.0 \times 10^{-6}$ & $2.1 \times 10^{-6}$ & $2.3 \times 10^{-6}$ & $2.1 \times 10^{-6}$ & $2.2 \times 10^{-6}$ \\
${ }_{\Lambda}^{5} \mathrm{He}$ & $3.1 \times 10^{-9}$ & $1.1 \times 10^{-9}$ & $2.4 \times 10^{-9}$ & $1.9 \times 10^{-9}$ & $7.7 \times 10^{-9}$ & $1.1 \times 10^{-8}$ & $6.6 \times 10^{-9}$ & $4.8 \times 10^{-9}$ \\
${ }_{\Lambda \Lambda}^{4} \mathrm{H}$ & $2.8 \times 10^{-7}$ & $1.5 \times 10^{-7}$ & $3.6 \times 10^{-7}$ & $4.1 \times 10^{-7}$ & $5.7 \times 10^{-7}$ & $7.7 \times 10^{-7}$ & $9.2 \times 10^{-7}$ & $1.3 \times 10^{-6}$ \\
${ }_{4 \Lambda}^{5} \mathrm{H}$ & $9.0 \times 10^{-10}$ & $3.1 \times 10^{-10}$ & $9.8 \times 10^{-10}$ & $9.4 \times 10^{-10}$ & $2.4 \times 10^{-9}$ & $4.4 \times 10^{-9}$ & $3.3 \times 10^{-9}$ & $3.6 \times 10^{-9}$ \\
${ }_{\Lambda \Lambda}^{5} \mathrm{He}$ & $9.0 \times 10^{-10}$ & $3.2 \times 10^{-10}$ & $9.9 \times 10^{-10}$ & $9.5 \times 10^{-10}$ & $2.4 \times 10^{-9}$ & $4.4 \times 10^{-9}$ & $3.3 \times 10^{-9}$ & $3.6 \times 10^{-9}$ \\
${ }_{4}^{6} \mathrm{He}$ & $2.1 \times 10^{-12}$ & $4.5 \times 10^{-13}$ & $1.9 \times 10^{-12}$ & $1.5 \times 10^{-12}$ & $7.5 \times 10^{-12}$ & $2.4 \times 10^{-11}$ & $8.6 \times 10^{-12}$ & $6.2 \times 10^{-12}$ \\
\hline
\end{tabular}


Table 4 Expected yields of some (hyper-)nuclei per event obtained by the advanced HRGM for the fit of ALICE data measured at $\sqrt{s_{N N}}=2.76$ $\mathrm{TeV}$ with different $\mathrm{CFO}$ scenarios. The $\mathrm{CFO}$ scenario is specified in parentheses

\begin{tabular}{lllllllll}
\hline Particle & $\begin{array}{l}\text { BMR EoS } \\
(\text { Single })\end{array}$ & $\begin{array}{l}\text { IST EoS } \\
(\text { Single })\end{array}$ & $\begin{array}{l}\text { BMR } \Lambda \text { EoS } \\
(\text { Single })\end{array}$ & $\begin{array}{l}\text { IST } \Lambda \text { EoS } \\
\text { (Single) }\end{array}$ & $\begin{array}{l}\text { BMR EoS } \\
\text { (Separate) }\end{array}$ & $\begin{array}{l}\text { IST EoS } \\
\text { (Separate) }\end{array}$ & $\begin{array}{l}\text { BMR } \Lambda \text { EoS } \\
\text { (Separate) }\end{array}$ & $\begin{array}{l}\text { IST } \Lambda \text { EoS } \\
(\text { Separate })\end{array}$ \\
\hline${ }^{3} \mathrm{H}$ & $2.3 \times 10^{-4}$ & $1.9 \times 10^{-4}$ & $2.3 \times 10^{-4}$ & $2.0 \times 10^{-4}$ & $3.4 \times 10^{-4}$ & $3.3 \times 10^{-4}$ & $3.4 \times 10^{-4}$ & $2.6 \times 10^{-4}$ \\
${ }_{\Lambda}^{4} \mathrm{H}$ & $4.0 \times 10^{-7}$ & $2.7 \times 10^{-7}$ & $3.8 \times 10^{-7}$ & $3.6 \times 10^{-7}$ & $1.2 \times 10^{-6}$ & $1.2 \times 10^{-6}$ & $1.0 \times 10^{-6}$ & $1.1 \times 10^{-6}$ \\
${ }_{\Lambda}^{4} \mathrm{He}$ & $4.0 \times 10^{-7}$ & $2.8 \times 10^{-7}$ & $3.8 \times 10^{-7}$ & $3.7 \times 10^{-7}$ & $1.2 \times 10^{-6}$ & $1.2 \times 10^{-6}$ & $1.0 \times 10^{-6}$ & $1.1 \times 10^{-6}$ \\
${ }_{\Lambda}^{5} \mathrm{He}$ & $5.3 \times 10^{-10}$ & $3.0 \times 10^{-10}$ & $4.6 \times 10^{-10}$ & $4.0 \times 10^{-10}$ & $2.8 \times 10^{-9}$ & $1.9 \times 10^{-9}$ & $2.1 \times 10^{-9}$ & $1.7 \times 10^{-9}$ \\
${ }_{4}^{4} \mathrm{H}$ & $9.2 \times 10^{-8}$ & $6.3 \times 10^{-8}$ & $1.1 \times 10^{-7}$ & $1.1 \times 10^{-7}$ & $3.1 \times 10^{-7}$ & $3.6 \times 10^{-7}$ & $3.8 \times 10^{-7}$ & $4.8 \times 10^{-7}$ \\
${ }_{\Lambda \Lambda}^{5} \mathrm{H}$ & $1.4 \times 10^{-10}$ & $7.8 \times 10^{-11}$ & $1.5 \times 10^{-10}$ & $1.4 \times 10^{-10}$ & $8.6 \times 10^{-10}$ & $7.0 \times 10^{-10}$ & $8.8 \times 10^{-10}$ & $9.1 \times 10^{-10}$ \\
${ }_{1}^{5} \mathrm{He}$ & $1.4 \times 10^{-10}$ & $7.9 \times 10^{-11}$ & $1.5 \times 10^{-10}$ & $1.4 \times 10^{-10}$ & $8.6 \times 10^{-10}$ & $7.1 \times 10^{-10}$ & $8.9 \times 10^{-10}$ & $9.2 \times 10^{-10}$ \\
${ }_{\Lambda \Lambda}^{6} \mathrm{He}$ & $1.6 \times 10^{-13}$ & $6.9 \times 10^{-14}$ & $1.5 \times 10^{-13}$ & $1.3 \times 10^{-13}$ & $1.7 \times 10^{-12}$ & $5.5 \times 10^{-13}$ & $1.5 \times 10^{-12}$ & $1.2 \times 10^{-12}$ \\
\hline
\end{tabular}

baryon chemical potential $[35,36]$ has a full-width at half maximum of about $40 \mathrm{MeV}$ of the continuum-extrapolated chiral susceptibility which peaks at the pseudocritical transition temperature $T_{c}=156.5 \pm 1.5 \mathrm{MeV}$ [61].

It is necessary to stress that the MHRGM worked out during last few years in [17-22] creates the new and rather high standards in the description of the experimental multiplicities of hadrons and light nuclear clusters. Moreover, this work proves once again that MHRGM based on the IST concept, is the most efficient, reliable and convenient tool to elucidate the subtleties of the CFO process in high energy nuclear collisions. Therefore, the question arises whether the successful phenomenology of the MHRGM based on the IST concept could find theoretical support by the microscopic approaches like the one suggested recently [30] in particular about the apparent independence of the hard core radii on temperature and density of the medium accessible in heavy ion collision experiments and about their dependence on the quark content of the hadrons involved. Furthermore, when in the future experiments at NICA JINR and FAIR GSI the accuracy of the collected data will be increased by an order of magnitude, the MHRGM based on the IST concept will be the key element of the phenomenological analysis because of its advantages over the other versions of the HRGM.

It is clear that, similarly to the usual chemistry, sooner or later the task of hadrochemistry to determine and to tabulate the classical second virial coefficients of all measured hadrons will be on demand. The IST EoS and its generalizations, which account for the curvature tension term [26,27], are perfectly suited for this task, since they allow one to model the high virial coefficients of classical and quantum systems with very high accuracy.

Furthermore, the examples of different mixtures studied in Ref. [27] by the EoS with the induced surface and curvature tensions give us a good hope that such EoS can be used to reliably model the mixtures of hadrons, nuclei and QGP bags, to elucidate the subtleties of interaction between the hadrons, nuclei and QGP bags and to study the phase transformations among them that occur in high energy nuclear collisions.

Acknowledgements The authors are thankful to Boris Grinyuk, Dmytro Oliinychenko and Ivan Yakimenko for the valuable comments. K.A.B. and G.M.Z. acknowledge support from the NAS of Ukraine by its priority project "Fundamental properties of the matter in the relativistic collisions of nuclei and in the early Universe" (No. 0120U100935). The work of L.V.B. and E.E.Z. was supported by the Norwegian Research Council (NFR) under grant No. 255253/F53 CERN Heavy Ion Theory, and by the RFBR grants No. 18-02-40085 and No. 18-0240084. K.A.B., O.V.V., and L.V.B. thank the Norwegian Agency for International Cooperation and Quality Enhancement in Higher Education for the financial support under grants CPEA-LT-2016/10094 and UTF-2016-long-term/10076. D.B.B. acknowledges partial support from RFBR under grant No. 18-02-40137 and from the National Research Nuclear University (MEPhI) in the framework of the Russian Academic Excellence Project under contract No. 02.a03.21.0005. The authors are grateful to the COST Action CA15213 "THOR" for supporting their networking. The ECT* Trento has supported this work and this infrastructure is part of a project that has received funding from the European Union's Horizon 2020 research and innovation programme under grant agreement No 824093.

Author contributions OVV suggested the main idea behind this work and together with $\mathrm{KAB}$ developed a novel formalism summarized in Eqs. (10)-(17). KAB together with DBB took the lead in writing the manuscript. OVV and ESZ performed fit of the experimental data on the light (anti)nuclei and hadrons under the supervision of LVB, and EEZ. OVV, ESZ, LVB and EEZ designed the figures. GMZ, LVB, EEZ contributed to the interpretation of the results and provided a critical feedback. All authors discussed the results and contributed to the final version of manuscript.

Data Availability Statement This manuscript has no associated data or the data will not be deposited. [Authors' comment: The data of this publication are available from the authors upon request.]

Open Access This article is licensed under a Creative Commons Attribution 4.0 International License, which permits use, sharing, adaptation, distribution and reproduction in any medium or format, as long as you give appropriate credit to the original author(s) and the source, provide a link to the Creative Commons licence, and indicate if changes were made. The images or other third party material in this article are included in the article's Creative Commons licence, unless indicated otherwise in a credit line to the material. If material is not 
included in the article's Creative Commons licence and your intended use is not permitted by statutory regulation or exceeds the permitted use, you will need to obtain permission directly from the copyright holder. To view a copy of this licence, visit http://creativecomm ons.org/licenses/by/4.0/.

\section{References}

1. Y. Cai, T.D. Cohen, B.A. Gelman, Y. Yamauchi, Phys. Rev. C 100, 024911 (2019). (and references therein)

2. D. Oliinychenko, talk given at XXVIIIth Conference "Quark Matter 2019“, arXiv:2003.05476v1 [hep-ph] (and references therein)

3. S. Mrówczyński, P. Słoń, Acta Phys. Polon. B 51, 1739 (2020)

4. S. Mrowczynski, arXiv:2004.07029v1 [nucl-th] (and references therein)

5. A. Andronic, P. Braun-Munzinger, K. Redlich, J. Stachel, Nature 561(7723), 321-330 (2018)

6. P. Braun-Munzinger, B. Dönigus, Nucl. Phys. A 987, 144 (2019)

7. R. Scheibl, U.W. Heinz, Phys. Rev. C 59, 1585 (1999)

8. J. Steinheimer, K. Gudima, A. Botvina, I. Mishustin, M. Bleicher, H. Stocker, Phys. Lett. B 714, 85 (2012)

9. D. Oliinychenko, L.G. Pang, H. Elfner, V. Koch, Phys. Rev. C 99, 044907 (2019)

10. S. Sombun et al., Phys. Rev. C 99, 014901 (2019)

11. STAR Collaboration (B. I. Abelev et al.), Science 328 (5974), 5862 (2010). https://doi.org/10.1126/science.1183980

12. STAR Collaboration (H. Agakishiev et al.), Nature 473(7347), 353-356 (2011). https://doi.org/10.1038/nature10079

13. STAR Collaboration (J. Adam et al.), Phys. Rev. C 99, 064905 (2019)

14. ALICE Collaboration (J. Adam et al.), Phys. Rev. C 93, 024917 (2016)

15. ALICE Collaboration (L. Ramonaet al.), AIP Conf. Proc. 1701(1), 080009 (2016)

16. ALICE Collaboration (J. Adam et al.), Phys. Lett. B 754, 360 (2016)

17. K.A. Bugaev et al., Phys. Part. Nucl. Lett. 15, 210 (2018)

18. K.A. Bugaev et al., Nucl. Phys. A 970, 133 (2018)

19. V.V. Sagun et al., Eur. Phys. J. A 54, 100 (2018)

20. K.A. Bugaev et al., J. Phys. Conf. Ser. 1390, 012038 (2019a)

21. B.E. Grinyuk et al. arXiv:2004.05481v1 [hep-ph] (2020)

22. K.A. Bugaev et al. arXiv:2005.01555v1 [nucl-th] (2020)

23. V.V. Sagun, A.I. Ivanytskyi, K.A. Bugaev, I.N. Mishustin, Nucl. Phys. A 924, 24 (2014)

24. K.A. Bugaev, A.I. Ivanytskyi, V.V. Sagun, E.G. Nikonov, G.M. Zinovjev, Ukr. J. Phys. 63, 863 (2018)

25. A.I. Ivanytskyi, K.A. Bugaev, V.V. Sagun, L.V. Bravina, E.E. Zabrodin, Phys. Rev. C 97, 064905 (2018)

26. K.A. Bugaev, Eur. Phys. J. A 55, 215 (2019)

27. N.S. Yakovenko, K.A. Bugaev, L.V. Bravina, E.E. Zabrodin, pp. 1-13. arXiv:1910.04889 [nucl-th]

28. W. Ebeling, D. Blaschke, R. Redmer, H. Reinholz, G. Röpke, J. Phys. A 42, 214033 (2009)

29. G. Röpke, D. Blaschke, T. Döppner, C. Lin, W.D. Kraeft, R. Redmer, H. Reinholz, Phys. Rev. E 99(3), 033201 (2019)
30. D. Blaschke, H. Grigorian, G. Röpke, Particles 2020(3), 477 (2020)

31. R. Venugopalan, M. Prakash, Nucl. Phys. A 546, 718 (1992)

32. L.M. Satarov, M.N. Dmitriev, I.N. Mishustin, Phys. Atom. Nucl. 72, 1390 (2009)

33. L.M. Satarov, K.A. Bugaev, I.N. Mishustin, Phys. Rev. C 91, $055203(2015)$

34. K.A. Bugaev et al., Universe 5, 00063 (2019)

35. Wuppertal-Budapest Collaboration (S. Borsanyi et al.), JHEP 1009, 073 (2010)

36. HotQCD Collaboration (A. Bazavov et al.), Phys. Rev. D 90, 094503 (2014)

37. K.A. Bugaev, Nucl. Phys. A 606, 559 (1996)

38. K.A. Bugaev, Phys. Rev. Lett. 90, 252301 (2003). (and references therein)

39. A. Andronic, P. Braun-Munzinger, J. Stachel, Nucl. Phys. A 772, 167 (2006). (and references therein)

40. D.R. Oliinychenko, K.A. Bugaev, A.S. Sorin, Ukr. J. Phys. 58, 211 (2013)

41. K.A. Bugaev, D.R. Oliinychenko, A.S. Sorin, G.M. Zinovjev, Eur. Phys. J. A 49, 30 (2013)

42. K.A. Bugaev et al., Europhys. Lett. 104, 22002 (2013)

43. V.V. Sagun, Ukr. J. Phys. 59, 755 (2014)

44. B. Dönigus, Int. J. Mod. Phys. E 29, 2040001 (2020a). (and references therein)

45. B. Dönigus, Eur. Phys. J. A 56, 280 (2020b). (and references therein)

46. J. Cleymans, S. Kabana, I. Kraus, H. Oeschler, K. Redlich, N. Sharma, Phys. Rev. C 84, 054916 (2011)

47. J. Stachel, A. Andronic, P. Braun-Munzinger, K. Redlich, J. Phys. Conf. Ser. 509, 012019 (2014)

48. J. Rafelski, Phys. Lett. B 62, 333 (1991)

49. A. Chodos, R.L. Jaffe, K. Johnson, C.B. Thorn, V.F. Weisskopf, Phys. Rev. D 9, 3471 (1974)

50. R. Hagedorn, Nuovo Cim. Suppl. 3, 147 (1965)

51. L.G. Moretto, K.A. Bugaev, J.B. Elliott, L. Phair, Europhys. Lett. 76, 402 (2006). (LBNL preprint 56898)

52. K.A. Bugaev, M. Gazdzicki, M.I. Gorenstein, Phys. Lett. B 523, $255(2001)$

53. K.A. Bugaev, J. Phys. G 28, 1981 (2002)

54. M.I. Gorenstein, K.A. Bugaev, M. Gazdzicki, Phys. Rev. Lett. 88, 132301 (2002)

55. K.A. Bugaev, M. Gazdzicki, M.I. Gorenstein, Phys. Lett. B 544, $127(2002)$

56. S. Chatterjee, R.M. Godbole, S. Gupta, Phys. Lett. B 727, 554 (2013)

57. S. Chatterjee et al., Adv. High Energy Phys. 2015, 349013 (2015)

58. K.A. Bugaev et al., Ukr. J. Phys. 61, 659 (2016)

59. A.J. Baltz et al., Phys. Lett. B 325, 7 (1994)

60. K.-J. Sun, L.-W. Chen, Phys. Rev. C 93, 064909 (2016)

61. A. Bazavov et al. [HotQCD], Phys. Lett. B 795, 15-21 (2019)

62. K. Gallmeister, C. Greiner, arXiv:2007.08258 [hep-ph] (2020) 